\title{
Virtual Humans' Behaviour: Individuals, Groups, and Crowds
}

\author{
Daniel Thalmann, Soraia Raupp Musse, Marcelo Kallmann \\ \{thalmann, soraia, kallmann\}@lig.di.epfl.ch \\ Computer Graphics Lab. Swiss Federal Institute of Technology \\ EPFL, DI-LIG, CH 1015 Lausanne, Switzerland
}

\begin{abstract}
In this paper, we first try to identify which mechanisms should be simulated in order to implement truly virtual humans or actors. We start from a structure linking perception, emotion, behavior, and action. Then, we emphasize the central concept of autonomy and introduce the concept of Levels of Autonomy. Finally, we propose a new abstraction for specification of behaviours in complex virtual environment simulations involving human agents, groups of agents, and interactive objects endowed with different levels of autonomy.
\end{abstract}

Keywords: virtual humans, intelligent agents, autonomous agents, crowd simulation, object interaction.

\section{Introduction}

Virtual human agents (here after just referred to as virtual humans, agents, or virtual actors) are humanoids whose behaviours are inspired by those of humans [1]. They can be equipped with sensors, memory, perception, and behavioural motor that allow them to act or react to events. They can also be much simpler, like guided by users in real time or interpreting predefined commands. The term group will be used to refer to a group of agents, and the term object for an interactive object of the environment. Agents, groups, and objects constitute the entities of the simulation.

A virtual actor inhabits a world which is dynamic and unpredictable. To be autonomous, it must be able to perceive its environment and decide what to do to reach the goal defined by its behavior. The relevant actions must then be transformed into motor control actions. Therefore, the design of a behavioral animation system raises questions about creating autonomous actors, endowing them with perception, selecting their actions, their motor control and making their behavior believable. They should appear spontaneous and unpredictable. They should give an illusion of life, making the audience believe that an actor is really alive and has its own will. As stated by Bates [2], believability of an actor is made possible by the emergence of emotions clearly expressed at the right moment. The apparent emotions of an actor and the way it reacts is what gives it the appearance of a living being with needs and desires. Without it, an actor would just look like an automaton. Moreover, the use of emotions make actors placed in the same context react differently. By defining different emergence conditions on different actors for their emotions, the generated emotions are ensured to be different and consequently derived behaviors are different.

A high-level behavioural autonomy concerns the ability to simulate complex behaviours. The most common method for simulation of simple acts of cognition is by using a rule-based system [3]. In this paper, we consider that the ability to simulate decision processes, memory, learning activities and performing specialised actions can be included in the agents (agents-based application), groups (groupsbased application) or in the objects (objects-based application).

Different parameters of agents' simulation can be defined in order to achieve the desired compromise between different requirements. Among others, interactivity, complex behaviours, intelligent abilities and frame rate of execution are directly related to the level of autonomy (LOA) that each entity of the simulation might have. Based on LOAS, we may then introduce three kinds of behavioural autonomy: guided, programmed and autonomous. Where guided represents the lower level of autonomy where the behaviours have to be informed by an external process (user, other system, etc). The programmed control implies to use a notation (language) to define possible behaviours, then the entity is able to translate this information in internal behaviours. The autonomous behaviour concerns the capability of acting independently exhibiting control over their internal state [4]. 


\section{Background Work}

The use of behavioral animation in generating computer animation is a well explored domain. Reynolds [5] described the first use of a behavioral model to produce a flocking behavior. Badler et al. [6] created a task planner and a biomechanical model for virtual humans illustrated with autonomous soldiers. Tu and Terzopoulos [7] created autonomous fishes living in a physically modeled virtual marine world. Hodgins et al. [8] described dynamic athletic behaviors. Unuma et al. [9] modeled human figure locomotion with emotions.

Other papers presented behavioral architectures. Brooks [10] [11] developed a subsumption architecture built in layers of behaviors. Maes [12] presented an action selection model where action selection is an emergent property of activation/inhibition dynamics among actions. Beer et al. [13] developed neural networks for insects. Ahmad et al. [14] described hierarchical concurrent state machines for behavior modeling and scenario control. Blumberg [15] [16] described an action selection model and created autonomous creatures.

Other papers presented interactive systems involving virtual creatures. Maes et al. [17] [18] designed a full-body interaction system with a virtual dog. Perlin [19] described virtual actors seemingly emotionally responsive using random noise functions.

Research in autonomy has also focused on social behaviors. Mataric [20] extended Brooks work to create complex group behavior produced from simple local interactions. Bécheiraz et al. [21] presented a model of nonverbal communication and interpersonal relationship between virtual actors. The Oz project [2] [22] [23] [24] focused on the creation of believable autonomous agents that exhibit rich personalities in interactive dramas.

Several works have discussed the various manners to simulate and interact with virtual agents. Zeltzer [25] presents a classification of levels of interaction and abstraction required in different applications. More recently, Thalmann et al, [26][27] proposed a new classification of synthetic actors according to the method of controlling motion, interaction and control of face and body. Noser and Thalmann [28] has described a L-system animation to model autonomous agents able to learn using synthetic vision and perception issues. Brogan et al [29] and Bouvier [30] have also presented groups and crowd simulations using particles systems and significant dynamic. In recent work, a crowd model has been introduced using different abstractions of behaviours, like the term guided crowd [31].

Kallmann et al [32] introduced the concept of smart-objects to specify entities containing interaction information of various kinds: intrinsic object properties, information on how-to-interact with it, objects functionality, and also expected agent behaviours. Applications based on agent-object interactions allow human factors engineers to test and analyse designed environments [33]. An example of such an application is presented by Johnson et al [34], whose purpose is to train equipment usage in a virtual environment.

\section{Modeling the Properties of Virtual Humans}

\subsection{Introduction}

For the modeling of actor behaviors, the ultimate objective is to build intelligent autonomous virtual humans with adaptation, perception and memory. These virtual humans should be able to act freely and emotionally. They should be conscious and unpredictable. But can we expect in the near future to represent in the computer the concepts of behavior, intelligence, autonomy, adaptation, perception, memory, freedom, emotion, consciousness, and unpredictability ? First, we will try to define these terms. More details may be found in [35].

- Behavior for virtual humans may be defined as a manner of conducting themselves. it is also the response of an individual, group, or species to its environment.

- Intelligence may be defined as the ability to learn or understand or to deal with new or trying situations.

- Autonomy is generally defined as the quality or state of being self-governing. 
- Adaptation: an artificial organism is adaptive as long as it may "survive" in more or less unpredictable and dangerous environments.

- Perception is defined as the awareness of the elements of environment through physical sensation.

- Memory is generally defined as the power or process of reproducing or recalling what has been learned and retained especially through associative mechanisms.

- Emotion may be defined as the affective aspect of consciousness; this is a state of feeling, a psychic and physical reaction subjectively experienced as strong feeling and physiologically involving changes that prepare the body for immediate vigorous.

- Consciousness may be defined as the quality or state of being aware especially of something within oneself or the state of being characterized by sensation, emotion, volition, and thought.

- Freedom for a virtual actor may be defined as the extent that his future behaviour is unpredictable to somebody.

In the next Sections, we will try first to identify which mechanisms should be simulated in order to implement truly virtual humans or actors. We can start from the structure proposed by Becheiraz and Thalmann [36] and shown in Figure 1. It links perception, emotion, behavior, and finally action. Then, we will discuss the important concepts of memory. Autonomy is a central characteristic that will be discussed in more details in Section 4, where Levels of Autonomy will be introduced. Unfortunately, other properties, like freedom and consciousness, or even intelligence, are still extremely hard to model. We will concentrate our efforts on behavior, autonomy, perception, memory, emotion, and action.

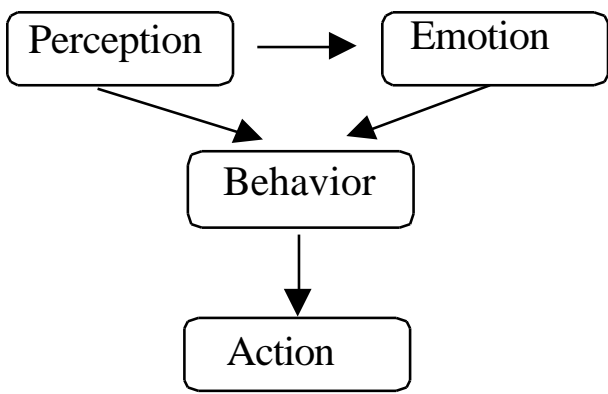

Figure 1. Structure of the behavioral model

\subsection{Perception}

Perception is defined as the awareness of the elements of environment through physical sensation. In order to implement perception, virtual humans should be equipped with visual, tactile and auditory sensors. These sensors should be used as a basis for implementing everyday human behaviour such as visually directed locomotion, handling objects, and responding to sounds and utterances. A simulation of the touching system should consist in detecting contacts between the virtual human and the environment. The most important perceptual subsystem is the vision system. A vision based approach for virtual humans is a very important perceptual subsystem and is for example essential for navigation in virtual worlds. It is an ideal approach for modeling a behavioral animation and offers a universal approach to pass the necessary information from the environment to the virtual human in the problems of path searching, obstacle avoidance, and internal knowledge representation with learning and forgetting characteristics. Visionbased behavioral models have been already described by Renault et al. [37] and Reynolds [38]. In [37], each pixel of the vision input has the semantic information giving the object projected on this pixel, and numerical information giving the distance to this object. So, it is easy to know, for example, that there is a table just in front at 3 meters. With this information, we can directly deal with the problematic question: "what do I do with such information in a navigation system?"

At a higher level, we may decompose perception as suggested by Becheiraz and Thalmann [36]. An actor's perception may include only the objects and the other actors in its neighborhood. But, in this case, it limits the possible behaviors because only the presence and the characteristics of an object or an actor are used to select a behavior. The actions of the actors and their consequences are not taken into account. The perception module should then produce three types of perception. The first type is the perception of the presence of objects and actors. It is called perception of objects. The second type is the perception of actions of actors. This perception is called perception of actions. The last type is the perception of actors performing actions on objects. It is called perception of events. The result of a perception is composed of 
three lists of objects, actions and events. Perception of events is slightly more complex because events themselves are decomposed into three classes: desirable events, events happening to another actor and potential events which may or may not occur. When the perception of an actor, one of its actions and the object involved together match the definition of a desirable event, it is perceived. The perception of an event happening to another actor is similar to that of desirable events, except that the actor is always different from the one perceiving the event. The perception of potential events is made in two steps. A potential event may actually occur after a certain time, or it may never happen. So, at first, a potential event is perceived and later it is either confirmed or unconfirmed. The perception of the nature and the characteristics of an object, an actor or an action is not easily done from their 3D representation. Recognizing an action through motion is difficult as well. The adopted solution is to categorize every object, actor and action based on its nature and characteristics.

\subsection{Emotion}

Emotion may be defined as the affective aspect of consciousness; this is a state of feeling, a psychic and physical reaction (as anger or fear) subjectively experienced as strong feeling and physiologically involving changes that prepare the body for immediate vigorous action virtual humans should be capable of responding emotionally to their situation as well as acting physically within it. Apart from making the virtual humans more realistic, visible emotions on the part of the virtual humans could provide designers with a direct way of affecting the user's own emotional state. Virtual humans will therefore be equipped with a simple computational model of emotional behaviour, to which emotionally related behaviour such as facial expressions and posture can be coupled, and which can be used to influence their actions.

An emotion is an emotive reaction of a person to a perception. This reaction induces him or her to assume a body response, a facial expression, a gesture or select a specific behavior. An emotion takes place between a perception and a subsequent reaction. Two different persons can thus have different reactions to the same perception according to the way they are affected by this perception.

Ortony et al. [39] describe an emotional model. The generated emotions belong to three classes which are the same as the perception classes. The emotions are generated in reaction to objects, actions of agents and events. The class of emotions caused by events is partitioned into three groups of emotion types. The first group concerns the emotions caused by potential events. The second group concerns events affecting the fortune of others and the last one concerns events affecting the well-being of the actor. Each class is characterized by emergence conditions for each of its emotions and variables affecting its intensity. The emotions felt by an actor are caused by its perception. Although some perceived objects, actors or actions are necessary for the emergence of an emotion, they may not possess some required qualities with sufficient intensity to produce an emotion effectively felt by the actor.

\subsection{Behavior}

Behavior is often defined as the way in which animals and humans act, and is usually described in natural language terms which have social, psychological or physiological significance, but which are not necessarily easily reducible to the movement of one or two muscles, joints or end effectors. Behavior is also the response of an individual, group, or species to its environment. Based on this definition, Reynolds [5] introduced the term and the concept of behavioral animation in order to describe the automating of such higher level animation. Behavior is not only reacting to the environment but should also include the flow of information by which the environment acts on the living creature as well as the way the creature codes and uses this information.

Behavior may be described in a hierarchical way. The behavioral model decomposes a behavior into simpler behaviors which may themselves be decomposed into other behaviors. Each level of this hierarchical decomposition contains one or more behaviors which are performed either sequentially, or concurrently. A level of the hierarchy containing several behaviors to be performed sequentially is called a behavior. Each behavior of a behavior sequence is called a behavioral cell. A behavioral cell contains behaviors which are performed either concurrently or exclusively when inhibition rules are specified. The behaviors contained in a behavioral cell are either behaviors or elementary behaviors. A behavior allows recursive decomposition in the hierarchy of a behavior. An elementary behavior is situated at the bottom of the hierarchical decomposition and encapsulates a specialized behavior which directly controls one or more actions. A behavior is executed by recursively performing each of the behavior, behavioral cell and elementary behavior entities at each level of the hierarchical structure of the behavior. At the top is a behavior entity with a finite state automata composed of at least one behavioral cell. The active behavioral cell is then performed. The state of each of its behaviors or elementary behaviors is evaluated. Then, for 
each active behavior or elementary behavior, the inhibition links, if any, are applied. Only active and noninhibited behaviors or elementary behaviors are performed. This process is applied recursively down to the bottom of the behavior hierarchy terminating with the elementary behaviors. The actions encapsulated by their specialized behaviors are then performed.

A high level behavior uses in general sensorial input and special knowledge. A way of modeling behaviors is the use of an automata approach. Each actor has an internal state which can change each time step according to the currently active automata and its sensorial input. In the following we use behavior and automata as synonyms. To control the global behavior of an actor we use a stack of automata. At the beginning of the animation the user provides a sequence of behaviors ( the script) and pushes them on the actor's stack. When the current behavior ends the animation system pops the next behavior from the stack and executes it. This process is repeated until the actor's behavior stack is empty. Some of the behaviors use this stack too, in order to reach subgoals by pushing itself with the current state on the stack and switching to the new behavior allowing them to reach the subgoal. When this new behavior has finished the automata pops the old interrupted behavior and continues. This behavior control using a stack facilitates to an actor to become more autonomous and to create his own subgoals while executing the original script.

\subsection{Action}

Based on perceptual information, his behavioral mechanism will determine the actions he will perform. Actions may be at several degrees of complexity. An actor may simply evolve in his environment or he may interact with this environment or even communicate with other actors. We will emphasize three types of actions: navigation and locomotion, grasping and ball games.

Actions are performed using a common architecture for motion [40]. The motion control part includes 5 generators: keyframing, inverse kinematics, dynamics, walking and grasping and high-level tools to combine and blend them. The action module [41] manages the execution of the different actions used by a behavior by animating a generic human model based on a node hierarchy [40]. It allows the concurrent or sequential execution of actions by managing smooth transitions between terminating and initiating actions.

The animation is driven by a behavioral loop. The role of the behavioral loop is to update the state of the virtual world corresponding to the new time. At each new iteration, the new time is incremented by a discrete time step. To update the state of the virtual world, the loop must update the state of each object and actor. In the case of an actor, the perception is first done, then its emotions are generated before its behavior and its actions are performed:

$$
\begin{gathered}
\text { repeat } \begin{array}{c}
\text { for each object and actor } \\
\text { perception }
\end{array} \\
\text { for each actor } \\
\text { emotions generation } \\
\text { for each object and actor } \\
\text { behavior execution } \\
\text { for each object and actor } \\
\text { actions execution }
\end{gathered}
$$

\subsection{Memory}

Memory is generally defined as the power or process of reproducing or recalling what has been learned and retained especially through associative mechanisms. This is also the store of things learned and retained from an organism's activity or experience as evidenced by modification of structure or behavior or by recall and recognition

To implement a concept of memory into a virtual human is not very complex, as already the memory is a key concept in Computer Science. For example, Noser and Thalmann [42] propose a dynamic occupancy octree grid to serve as a global 3D visual memory and to allow an actor to memorize the environment that he sees and to adapt it to a changing and dynamic environment. His reasoning process allows him to find 3D paths based on his visual memory by avoiding impasses and circuits. The global behavior of the actor is based on a navigation automata, representing the automata of an actor, who has to go from his current position to different places, memorized in a list of destinations. He can displace himself in known or unknown environments. 


\section{Autonomy and Levels of Autonomy}

\subsection{Autonomy}

Autonomy is generally defined as the quality or state of being self-controlled. Bourgine [43] defines an autonomous system as a system which has the abdictive capacity to guess viable actions. As stated by Courant et al. [44], in cybernetics as well as in cognitive psychology, autonomy has always been strongly connected with self-organization. Hence, computer scientists sometimes prefer to take the following definition of autonomy "the capacity of a system to maintain its viability in various and changing environments".

The need to have autonomous behaviour for virtual humans arises from several considerations:

- in Virtual Environments where users can have aid from Virtual Humans for learning, equipment training etc.

- in computer-generated films, the more autonomous behaviour that is built into the virtual humans, the less extra work there is to be done by the designer to create complete scenarios

- in simulation of real-life events, like building evacuation in case of fire etc.

- in interactive games, autonomous human-like behaviour is necessary in order to maintain the illusion in the user that the Virtual Humans are real ones.

Different parameters of agents' simulation can be defined in order to achieve the desired compromise between different requirements. Among others, interactivity, complex behaviours, intelligent abilities and frame rate of execution are directly related to the level of autonomy (LOA) that each entity of the simulation might have. Based on the Level of Autonomy (LOA), we may then introduce three kinds of behavioural autonomy: guided, programmed and autonomous. Where guided represents the lower level of autonomy where the behaviours have to be informed by an external process (user, other system, etc). The programmed control implies to use a notation (language) to define possible behaviours, then the entity is able to translate this information in internal behaviours. Yet, guided or programmed agents can also be useful depending on the application. When less compromise with complex behaviours is necessary in the simulation, agents that are "less autonomous" can represent a best performance in terms of execution frame-rate and interactivity. Table 1 presents some comparison data between these three kinds of LOA.

\begin{tabular}{||l|l|l|l||}
\hline & GUIDED & PROGRAMMED & AUTONOMOUS \\
\hline Memory & $\begin{array}{l}\text { Generally not } \\
\text { provided }\end{array}$ & $\begin{array}{l}\text { Generally not } \\
\text { provided }\end{array}$ & $\begin{array}{l}\text { Connected with others parameters } \\
\text { internally to agents }\end{array}$ \\
\hline Learning & Not provided & Not provided & Can be present \\
\hline Autonomy & Low & Medium & High \\
\hline Self-control & Not provided & Not provided & $\begin{array}{l}\text { Result of a behavioural complex } \\
\text { process using other internal } \\
\text { parameters }\end{array}$ \\
\hline Perception & $\begin{array}{l}\text { Generally not } \\
\text { provided }\end{array}$ & $\begin{array}{l}\text { Should be } \\
\text { provided } \\
\text { connected to other parameters, e.g. } \\
\text { action. }\end{array}$ \\
\hline Behaviour & Driven-oriented & Program-oriented & $\begin{array}{l}\text { Agent-oriented. Result of } \\
\text { behavioural process using other } \\
\text { parameters, e.g. perception. }\end{array}$ \\
\hline Action & Driven-oriented & Program-oriented & Agent-oriented decision. \\
\hline Motion & Driven-oriented & Program-oriented & Motion planning based. \\
\hline
\end{tabular}

Table 1: Comparison between different LOAs

The classification presented in Table 1 shows the mainly difference between the three kinds of agent control. Anyway, some different systems can be developed mixing the control in a same simulation. For instance, an agent could have a programmed or guided motion, but also memory with learning processes in order to achieve new behaviours, which can have more priority than the programmed or guided behaviours. 
Table 2 exemplifies the three kinds of agent autonomy using two different agent tasks: i) agent goes to a specific location; ii) agent applies specific action.

\begin{tabular}{|l|l|l|}
\hline Agent LOA & \multicolumn{1}{|c|}{ Agent goes to a specific location } & \multicolumn{1}{|c|}{ Agent applies a specific action } \\
\hline Guided & $\begin{array}{l}\text { Agent needs to receive during the } \\
\text { simulation a list of collision-free } \\
\text { positions }\end{array}$ & $\begin{array}{l}\text { Agent needs to receive information } \\
\text { about the action to be applied }\end{array}$ \\
\hline Programmed & $\begin{array}{l}\text { Agent is programmed to manage the } \\
\text { information of a path to follow while } \\
\text { avoiding collision with other agents } \\
\text { and programmed obstacles }\end{array}$ & $\begin{array}{l}\text { Agent is programmed to manage } \\
\text { where and how the action can } \\
\text { occur }\end{array}$ \\
\hline Autonomous & $\begin{array}{l}\text { Agent is able to perceive information } \\
\text { in the environment and decide a path } \\
\text { to follow to reach the goal, using the } \\
\text { environment perception or the memory } \\
\text { (past experiences) }\end{array}$ & $\begin{array}{l}\text { Agent can decide about an action } \\
\text { to be applied. This action can be } \\
\text { programmed, imitated or existent in } \\
\text { the memory (past experiences) }\end{array}$ \\
\hline
\end{tabular}

Table 2: LOA present in different agent-oriented tasks.

\subsection{LOA Related to Groups of Agents}

In the case of crowd simulation, usually we intend to have lots of virtual human agents avoiding dealing with individual behaviours. Contrary to the last section, our goal here is to describe methods to provide intelligence focused in common group entity that controls its individuals. Figure 2 shows the co-relation between these two parameters.

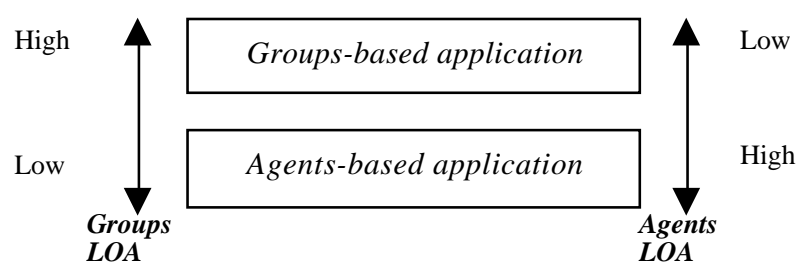

Figure 2: Co-relation between groups and agents LOA.

We have called groups-based application, the crowd and group applications, where individual complexity is less required. In this case, the intelligence abstraction can be included in the groups providing more autonomy to the groups instead to the individuals. On the other hand, agents-based applications concern more complex individuals as showed in the last section. Some different classes of techniques have been discussed in the literature depending on the number of elements being controlled and the sophistication of the control strategy [3]: Particle [45], Flocking [5] and Hybrid systems, mixing particle, flocking and reactive behaviours [31].

\begin{tabular}{|c|c|c|c|} 
Method & $\underline{\text { Particle systems }}$ & $\underline{\underline{\text { Flocking }}}$ & $\underline{\underline{\text { Hybrid }}}$ \\
\hline$\underline{\text { Structurems }}$ & $\begin{array}{c}\text { Non- } \\
\text { hierarchical }\end{array}$ & $\begin{array}{c}\text { Levels: } \\
\text { flock, agents }\end{array}$ & $\begin{array}{c}\text { Levels: } \\
\text { Crowd, } \\
\text { groups, } \\
\text { agents }\end{array}$ \\
\hline$\underline{\text { Participants }}$ & many & Some & Many \\
\hline$\underline{\text { Intelligence }}$ & none & Some & Some \\
\hline$\underline{\text { Physics- }}$ & yes & Some & Can be \\
\hline$\underline{\text { Collision }}$ & Detect & Avoidance & Avoidance \\
\hline$\underline{\text { Control }}$ & Force fields & $\begin{array}{c}\text { Local } \\
\text { tendency in } \\
\text { the flock } \\
\text { structure }\end{array}$ & $\begin{array}{c}\text { Pre-defined, } \\
\text { rules and } \\
\text { guided }\end{array}$ \\
\hline
\end{tabular}

Table 3: Table modified from the original [3] in order to include Hybrid systems [31] 
The methods presented in Table 3 are characterised by emergent behaviours: a global effect generated by local rules [3]. Although some crowd simulations seem to be formed by autonomous agents, this is not completely true. In fact, normally it concerns simple individuals controlled by complex groups' behaviours. However, some rule-based behavioural animation can be used in simulations formed by small groups as characterised in Table 4 [3].

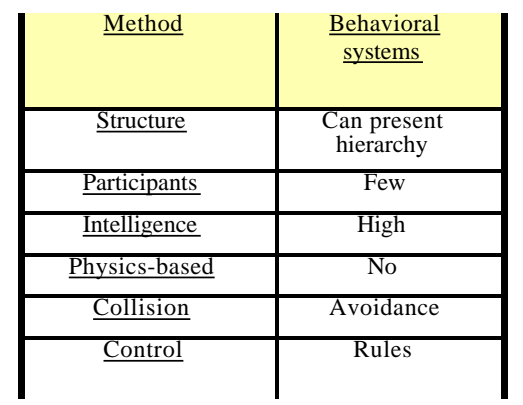

Table 4: Behavioural method to simulate complex behaviours

Considering levels of autonomy (LOA), we have classified the crowd behaviours in three kinds:

i) Guided crowds, which behaviours are defined explicitly by the users;

ii) Programmed crowds, which behaviours are programmed in a script language;

iii) Autonomous crowds, which behaviours are specified using rules or others complex methods.

Table 5 exemplifies this classification of crowd autonomy using two different crowd tasks: "group goes to a specific location", and "group reacts to matched event".

\begin{tabular}{|l|l|l|}
\hline Crowd LOA & Group goes to a specific location & \multicolumn{1}{|c|}{ Group reacts to matched event } \\
\hline Guided & $\begin{array}{l}\text { Group needs to receive during the } \\
\text { simulation a list of positions "in- } \\
\text { betweens" in order to reach the } \\
\text { goal }\end{array}$ & $\begin{array}{l}\text { Group needs to receive an } \\
\text { information about the matched } \\
\text { event and the reaction to be } \\
\text { applied }\end{array}$ \\
\hline Programmed & $\begin{array}{l}\text { Group is programmed to manage the } \\
\text { information of a path to follow } \\
\text { avoiding collision with other } \\
\text { agents and programmed obstacles. }\end{array}$ & $\begin{array}{l}\text { Group can manage events and } \\
\text { reactions, which are programmed. }\end{array}$ \\
\hline Autonomous & $\begin{array}{l}\text { Group is able to perceive } \\
\text { information in the environment and } \\
\text { decide a path to follow to reach the } \\
\text { goal, using the environment } \\
\text { perception or the memory (past } \\
\text { experiences). }\end{array}$ & $\begin{array}{l}\text { Group can perceive a matched } \\
\text { event and decide about the } \\
\text { reaction to be applied. This } \\
\text { reaction can be also programmed or } \\
\text { existent in the group memory (past } \\
\text { experiences). }\end{array}$ \\
\hline
\end{tabular}

Table 5: LOA present in different group-oriented tasks

Considering the hierarchy existent normally in crowds systems (crowds, groups and agents), complex structures like memory, decision and etc, can be defined in the group level, in this case optimising the fact that the agents do not need to have these information.

\subsection{LOA Related to Objects}

Whenever the simulation needs to handle complex agent-object interactions, many difficult issues arise. Such difficulties are related to the fact that each object has its own movements, functionality and purposes.

There is a range of growing complexity for possible agent-object interactions in a virtual environment. Examples are actions like grasping a fruit, automatic doors that open when agents are nearby and also complex ones like entering a lift. 
One can consider that agents' perceptions can solve all the necessary reasoning and planning processes to achieve some simple tasks, as for instance a single-hand automatic grasping of small objects [46]. But this is no more possible for interactions with objects that have an intricate proper functionality, as the lift example (Figure 6). Moreover, even for simpler interactions as our grasping example, we did not consider semantic aspects, e.g. recognising through sensors if a given fruit can be eaten or not.

A first approach to overcome these difficulties is to maintain a table with some semantic and grasping information for all graspable objects [47]. Another approach models all possible object interaction features like its functionality and semantic information, containing also a complete description of all possible interactions it can offer to the agent [32].

In fact, each time more information related to the object is given, its level of autonomy (LOA) is increased. In the scope of simulations in virtual environments, increasing the LOA of an object, will make it move from a guided state through a programmed state until achieving a complete autonomous state. In the lowest LOA, the object only knows possible movements to be applied to its parts. In the highest LOA, the object has all interaction information necessary, in a form of pre-defined plans, to take control over the agent to make it perform the interaction. In a mid-term, the programmed object controls its moveable parts, based on the agent decisions taken during the interaction.

Table 6 illustrates how an agent must proceed according to the different LOAs for three different interactive objects of the environment: a door that opens with a simple lateral translation movement, a direction sign, and a two-stage lift.

\begin{tabular}{|c|l|l|l|}
\hline Object LOA & \multicolumn{1}{|c|}{ Door } & \multicolumn{1}{c|}{ sign } & \multicolumn{1}{c|}{ Lift } \\
\hline Guided & $\begin{array}{l}\text { The agent have to move } \\
\text { its arm to a attainable } \\
\text { and meaning location of } \\
\text { the door, and control its } \\
\text { movement until open it. }\end{array}$ & $\begin{array}{l}\text { The agent recognises } \\
\text { that the sign has an } \\
\text { arrow and recognises } \\
\text { the showed direction. }\end{array}$ & $\begin{array}{l}\text { The agent recognises where } \\
\text { is the call button, how and } \\
\text { when the door opens, how } \\
\text { and where to enter inside the } \\
\text { lift, when and how to go out, } \\
\text { etc. }\end{array}$ \\
\hline Programmed & $\begin{array}{l}\text { The agent has to move } \\
\text { its arm to the right place } \\
\text { but the door opens by } \\
\text { itself. }\end{array}$ & $\begin{array}{l}\text { The agent recognises } \\
\text { the sign, but the } \\
\text { direction is given with } \\
\text { no recognition. }\end{array}$ & $\begin{array}{l}\text { The agent accesses the } \\
\text { current lift state and decides } \\
\text { only its moves accordingly. }\end{array}$ \\
\hline Autonomous & $\begin{array}{l}\text { The door takes control of } \\
\text { the agent telling exactly } \\
\text { the place to put its hand } \\
\text { and the complete } \\
\text { movement of the door }\end{array}$ & $\begin{array}{l}\text { The sign gives a new } \\
\text { direction to go for each } \\
\text { agent that passes } \\
\text { nearby. }\end{array}$ & $\begin{array}{l}\text { The lift takes control of the } \\
\text { movements of the agent and } \\
\text { gives him a complete plan, } \\
\text { based on primitive actions, to } \\
\text { perform the interaction. }\end{array}$ \\
\hline
\end{tabular}

Table 6: LOA present in different objects-oriented tasks

Depending on the LOA of each object, different sensors (see table 1) are required to exist in the agent in order to perform an interaction. Such sensors can be difficult to control and expensive in terms of both computer memory and computer process time. To minimise such side effects, and depending on the application, it can be interesting to use highly autonomous interactive objects. That means to adopt the strategy of leaving inside each interactive object a list of available pre-defined plans that are automatically updated depending on objects' internal states.

\subsection{A New Abstraction for Specification of Behaviours}

As presented in the last sections, our new paradigm to define the LOA of virtual human agents considers that the "intelligence" is not only included in the virtual human agents, but can be also included in groups and objects. In this section, we describe this new paradigm mentioning examples of different simulations where the autonomy abstraction is variable for the entities of the simulation. Considering the abstraction levels: guided, programmed and autonomous behaviours, we present a schema that includes the entities group and object, as showed in Figure 3. 


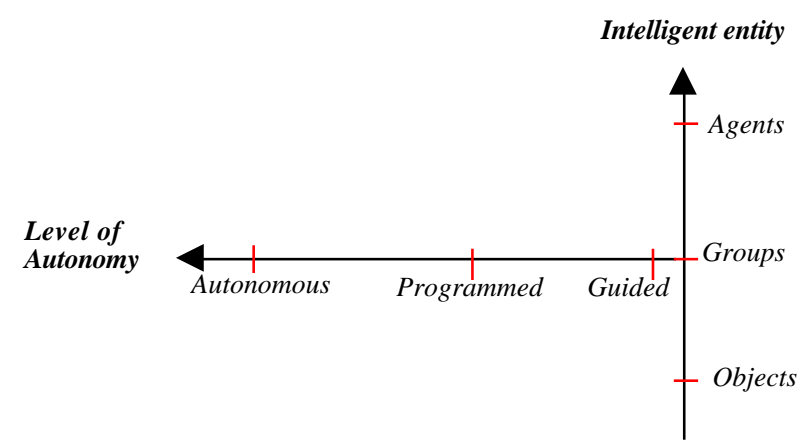

Figure 3: Level of autonomy vs. intelligent entity.

We can so classify a simulation in terms of the autonomy distribution among its entities, i.e., a simulation (Si), can be translated as a function of three components: agents, groups and objects, as follows:

$$
\mathrm{S}_{\mathrm{i}}=\mathrm{f}(\mathrm{LOA}(\text { Agents }), \text { LOA(Groups), LOA(Objects) })
$$

In this way, depending on the application, one can choose the best distribution of autonomy to adopt. In general, if the simulation wants to focus in the behaviour of a given entity, this entity might have a maximum LOA.

Interesting cases may arise when we choose to have different LOAs among the individuals of a same entity type. For example, consider the case of a simulation of autonomous agents, with a limited set of sensors, interacting with objects. The objects of the environment which are simple enough to be guided by such agents can be initialised as guided; while other more complex objects can be initialised with more autonomy. A consistent strategy of priorities and negotiation must be adopted in simulations where two entities with high LOA need to achieve a common task. One example is when an autonomous agent receives from an autonomous object a complete plan to achieve some interaction task. In this case, the agent will use its sensors to validate, or even ameliorate, the given plan. For this to happen, both entities must be capable to negotiate having a common notion of priorities. A similar negotiation is needed when an autonomous agent is required to follow a behaviour that comes from its current autonomous group control.

These simulations are examplified with the following description of three simulations:

i) Simulation of autonomous agents in a train station, which involves interaction with several objects: chairs, counters and a lift. Most of them are guided objects while the lift, which has a complex functionality, is autonomous. Thus, we consider that the overall object autonomy is medium (programmed). The agent can perceive and interact with the different kinds of objects, to sit down into the chair, buy a ticket in a counter and taking the lift. Although, due to the limited set of agent perceptions, no negotiation is done between the agent and the lift, the agent just accepts the autonomous behaviour of the lift (figure 4). 


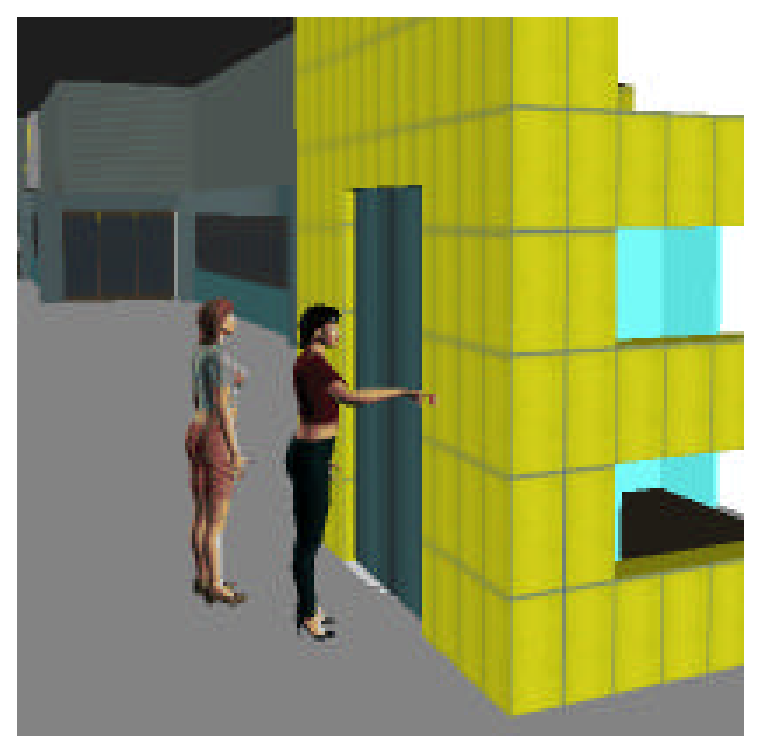

Figure 4: Agents interacting with a lift. Image generated using a lift programmed as a smart object [10].

ii) Simulation of groups of agents involved in a political demonstration. The groups have their motion programmed but are autonomous concerning their perception of other agents and their ability to react to the proximity of others (figure 5). As in the example i) we chosen to represent this mixed control as medium LOA. The agents are just programmed according to the groups' behaviours.

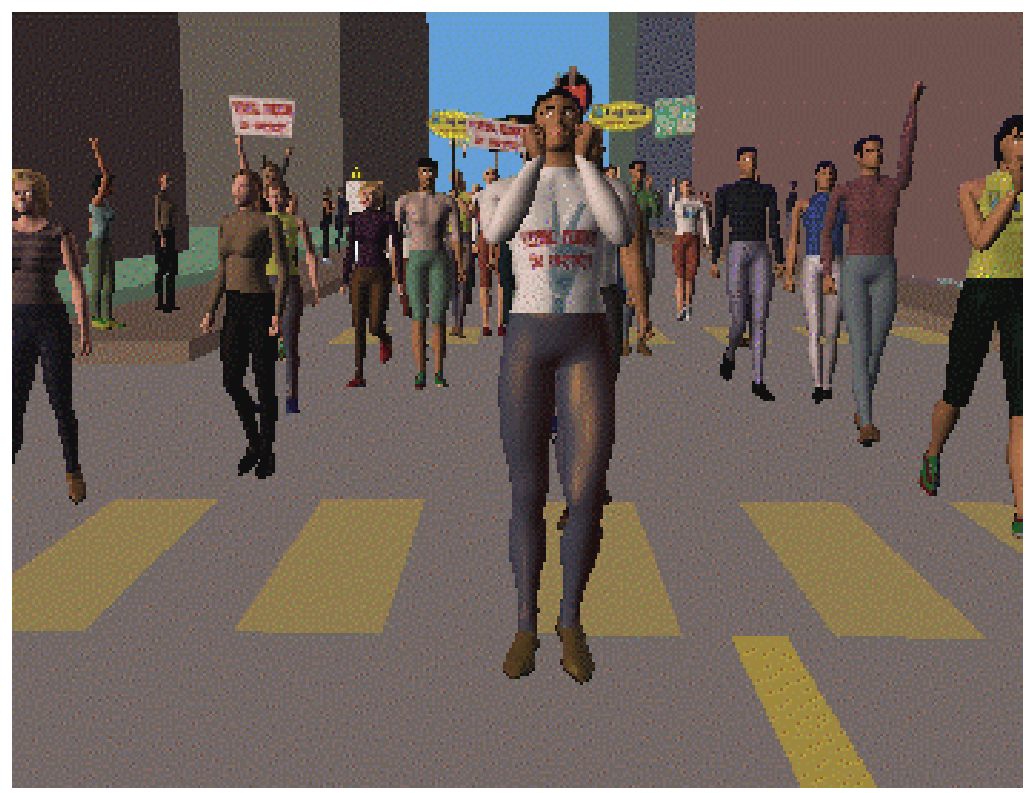

Figure 5: Political demonstration where autonomous and programmed groups are able to interact with others and react as a function of it. Image generated using a crowd simulation framework [16].

iii)Simulation of a party populated by autonomous groups. The groups have two possible emotional status: SOCIAL (representing groups that prefer to walk and meet others than eating) or HUNGRY (the opposite idea). Trough the meeting of groups, the emotional status can be dynamically changed as a function of sociological rules [48]. If a group decides to eat something, the autonomous object table [32] controls the interaction with the table of food (figure 6). 


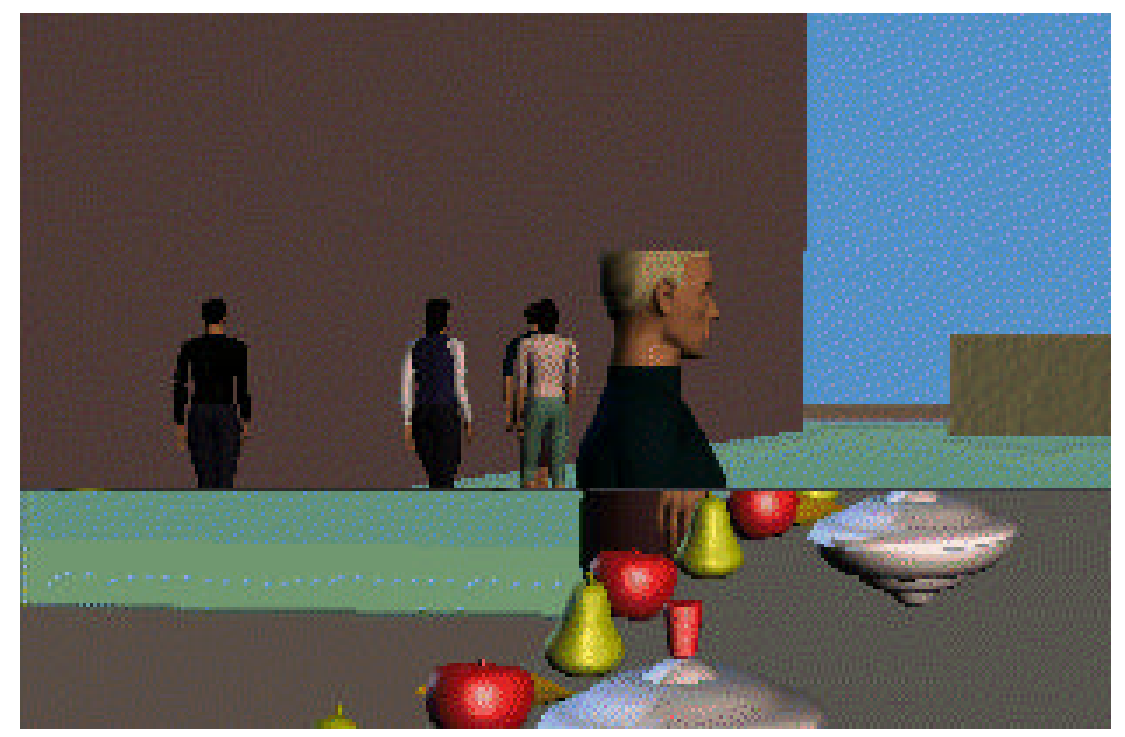

Figure 6: Autonomous groups interacting with autonomous objects. Image generated mixing a smart object control [32] inside the crowd simulation framework [31].

\section{Conclusions}

We proposed in this paper a new abstraction to be considered in order to distribute the autonomy among the entities of the simulation. The idea we dealt here concerns the possibility to improve the frame rate of execution as well as to optimise the complexity required, by distributing some knowledge and autonomy to others entities of the simulation: groups and objects. This paradigm has been tested in the context of a Virtual City project [49] because we have to simulate several virtual human agents that can act in differently ways and apply different actions.

\section{Acknowledgements}

The research was sponsored by the Swiss National Research Foundation, FUNDEPE, CAPES and CNPq (Brazilian offices of Education and Science).

\section{References}

1 Meyer, J.A., Guillot, A. "From SAB90 to SAB94: Four Years of Animat Research". In: Proceedings of Third International Conference on Simulation of Adaptive Behavior. Brighton, England, 1994.

2 Bates, J.. "The Role of Emotion in Believable Agents. In Communication of the ACM. Vol. 37, No. 7, pages 122-125, July 1994. Parent, R. "Computer Animation: Algorithms and Techniques, http://www.cis.ohiostate.edu/ parent/OxfordPress.html.

4 Wooldridge, M. and Jennings, N. "Intelligent Agents: Theory and Practice". Knowledge Engineering Review, 10(2), June 1995. Cambridge University Press, 1995.

5 Reynolds C. (1987) "Flocks, Herds, and Schools: A Distributed Behavioral Model", Proc.SIGGRAPH '87, Computer Graphics, Vol.21, No4, pp.25-34

6 Badler, N.I., Phillips, C., and Webber, B.L.. "Simulating Humans: Computer Graphics, Animation, and Control". 1993, Oxford University Press, New York.

7 Tu X., Terzopoulos D. (1994) "Artificial Fishes: Physics, Locomotion, Perception, Behavior", Proc. SIGGRAPH '94, Computer Graphics, pp.42-48.

8 Hodgins, J.K., Wooten, W.L., Brogan D.C., O'Brien, J.F.. "Animating Human Athletics". Proceedings of SIGGRAPH 95, p. 71-78 (Los Angeles, CA, August 6-11, 1995).

9 Unuma, M., Anjyo, K., Takeuchi, R. "Fourier Principles for Emotion-based Human Figure Animation". Proceedings of SIGGRAPH 95, p. 91-96 (Los Angeles, CA, August 6-11, 1995). 
Brooks, R.A.. “A Robust Layered Control System for a Mobile Robot. IEEE Journal of Robotics and Automation. Volume RA-2, Number 1, 1986.

11 Brooks, R.A.. "A Robot That Walks; Emergent Behaviors from a Carefully Evolved Network. MIT AI Lab Memo 1091, February 1989.

12 Maes, P.. "How to Do the Right Thing, Connection Science, Vol. 1, No. 3, 1989

13 Beer, R.D.., Ritzmann, R.E. and McKenna, T., Eds. "Biological Neural Networks in Invertebrate Neuroethology and Robotics". Academic Press, 1993.

14 Ahmad, O., Cremer, J., Hansen, S., Kearny, J., and Willemsen, P. "Hierarchical, Concurrent State Machines for Behavior Modeling and Scenario Control". In Conference on AI, Planning, and Simulation in High Autonomy Systems, Gainesville, Florida, Usa, 1994.

Blumberg, B.M.. "Action-Selection in Hamsterdam: Lessons from Ethology”. In Third International Conference on the Simulation of Adaptive Behavior, Pages 108-117, Brighton, England, 1994. Blumberg, B.M. and Galyean, T.A,. "Multi-level direction of Autonomous Creatures for Real-Time Vitual Environment". In Proceedings of SIGGGRAPH 95, pages 47-54, August 1995.

17 Maes, P.. "Artifical Life Meets Entertainment: Lifelike Autonomous Agents". In Communication of the ACM. Vol. 38, No. 11, pages 108-114, November 1995.

18 Maes, P., Darell, T., Blumberg, B., Pentland, A.. "The ALIVE System: Full-Body Interaction with Autonomous Agents". Proceedings of Computer Animation 95, p. 11-18 (Geneva, Switzerland, April 19-21, 1995).

19 Perlin K.. "Interacting with virtual actors". Visual Proceedings of SIGGRAPH 95, p. 92-93 (Los Angeles, CA, August 6-11, 1995).

20 Mataric. M.J. "From Local Interactions to Collective Intelligence". From the book: The biology and Technology of Intelligent Autonomous Agents. Edited by Luc Steels. NATO ASI Series F, p. 275295, Vol. 144, 1995.

21 Bécheiraz, P. and Thalmann, D.. "A Model of Nonverbal Communication and Interpersonal Relationship between Virtual Actors". In Proceedings of Computer Animation 96, Geneva, 1996

22 W. S. N. Reilly. "Believable Social and Emotional Agents". Technical Report CMU-CS-96-138, School of Computer Science, Carnegie Mellon University, December 1996.

23 Loyall, A.B. and Bates, J. "Personality-Rich Believable Agents That Use Language". Proceedings of the First International Conference on Autonomous Agents, February 1997, Marina del Rey, California.

24 Mateas, M, “An Oz-Centric Review of Interactive Drama and Believable Agents". Technical Report CMU-CS-97-156, School of Computer Science, Carnegie Mellon University, Pittsburgh, PA. June 1997.

25 Zeltzer, D. "Task-level Graphical Simulation: Abstraction, Representation and Control”. Making them Move: Mechanics, Control and Animation of Articulated Figures. Edited by N. Badler, B. Barsky and D. Zeltzer. Pp 3-33. 1991.

26 Thalmann, D., "A New Generation of Synthetic Actors: The Interactive Perceptive Actors". Proc. Pacific Graphics 96, National Chiao Tung University Press, Hsinchu, Taiwan, 1996, pp. 200 219.

27 Cavazza, M., Earnshaw, R., Magnenat-Thalmann, N. and Thalmann, D. "Motion Control of Virtual Humans". IEEE CG\&A, Vol. 18, No. 5, Sept/Oct 1998, pp.24-31..

28 Noser H. and Thalmann D., (1996) The Animation of Autonomous Actors Based on Production Rules, Proceedings Computer Animation'96, June 3-4, 1996, Geneva Switzerland, IEEE Computer Society Press, Los Alamitos, California, pp 47-57

29 Brogan, D.C., Metoyer, R.A. and Hodgins, J.K. "Dynamically simulated characters in virtual environments". In IEEE COmputer Graphics and Applications. Vol.18, No5, pp 58-69. Sept. 1998.

30 Bouvier, E.; Cohen E.; and Najman. L. "From crowd simulation to airbag deployment: particle systems, a new paradigm of simulation". Journal of Electronic Imaging 6(1), 94-107 (January 1997).

31 Musse, S.R., Babski, C., Capin, T. and Thalmann, D. "Crowd Modelling in Collaborative Virtual Environments". ACM VRST /98, Taiwan

32 Kallmann, M. and Thalmann, D. "Modeling Objects for Interaction Tasks", Proc. Eurographics Workshop on Animation and Simulation, 1998.

33 Badler, N. "Virtual Humans for Animation, Ergonomics, and Simulation", IEEE Workshop on Non-Rigid and Articulated Motion, Puerto Rico, June 97.

34 Johnson, W. L. and Rickel, J. "Steve: An Animated Pedagogical Agent for Procedural Training in Virtual Environments", Sigart Bulletin, ACM Press, vol. 8, number 1-4, 16-21, 1997. 
Magnenat Thalmann N., Thalmann D. "Creating Artificial Life in Virtual Reality" in: (Magnenat Thalmann and Thalmann, eds) Artificial Life and Virtual Reality, John Wiley, Chichester, 1994, pp.1-10

Becheiraz, P. and Thalmann, D. "A Behavioral Animation System for Autonomous Actors personified by Emotions", Proc. First Workshop on Embodied Conversational Characters (WECC 98), Lake Tahoe, USA.

37 Renault, O., Magnenat-Thalmann, N. Thalmann, D. "A Vision-based Approach to Behavioral Animation", The Journal of Visualization and Computer Animation, Vol. 1, No. 1, pp. $18-21$. Reynolds CW (1993) An evolved, Vision-Based Behavioral Model of Coordinated Group Motion, in: (Meyer JA, Roitblat HL, Wilson SW, eds) From Animals to Animats, Proc. 2nd International Conf. on Simulation of Adaptive Behavior, MIT Press.

39 Ortony, A., Clore G. L. and Collins, A.. The Cognitive Strucutre of Emotions. Cambridge University Press, 1990.

40 Boulic R., Capin T., Kalra P., Lintermann B., Moccozet L., Molet T., Huang Z., MagnenatThalmann N., Saar K., Schmitt A., Shen J. and Thalmann D. The HUMANOID Environment for Interactive Animation of Multiple Deformable Human Characters. Proceedings of EUROGRAPHICS 95, p. 337-348 (Maastricht, The Netherlands, August 28-September 1, 1995).

41 R. Boulic, P. Bécheiraz, L. Emering and D. Thalmann. Integration of Motion Control Techniques for Virtual Human and Avatar Real-Time Animation. In Proc. VRST '97, ACM Press, pages 111118, September 1997.

42 Noser H., Renault O., Thalmann D., Magnenat Thalmann N. (1995) "Navigation for Digital Actors based on Synthetic Vision, Memory and Learning", Computers and Graphics, Pergamon Press, Vol.19, No1, pp.7-19.

43 Bourgine P (1994) Autonomy, Abduction, Adaptation, in: (Magnenat Thalmann N, Thalmann D, eds), Proc. Computer Animation '94, IEEE Computer Society Press.

44 Courant M, Beat Hirsbrunner B, Stoffel B (1994) Managing Entities for an Autonomous Behaviour, in: This Volume.

45 Reeves, W. "Particle Systems - A Technique for Modeling a Class of Fuzzy Objects", ACM Transactions on graphics - April, 1993 - Vol. 2, No. 2.

46 Huang, Z., et al. "A Multi-sensor Approach for Grasping and 3D interaction", Proc. Computer Graphics international'95, Leeds, Academic Press, pp. 235-254.

47 Levison, L. "Connecting Planning and Acting via Object-Specific reasoning", PhD thesis, Dept. of Computer \& Information Science, University of Pennsylvania, 1996.

48 Musse, S.R. and Thalmann, D. "A Model of Human Crowd Behavior: Group Inter-Relationship and Collision Detection Analysis". Proc. Workshop of Computer Animation and Simulation of Eurographics'97, Sept, 1997. Budapest, Hungary.

49 Farenc, N; et al. "A Paradigm for Controlling Virtual Humans in Urban Environment Simulation". Applied Artificial Intelligence Journal. Special issue on Artificial Intelligence. 1999 (to appear) 